\title{
Pathological investigation and viral antigen distribution of emerging African Swine Fever in Vietnam
}

\author{
Uda Zahli Izzati ${ }^{1}$, Minori Inanaga ${ }^{1}$, Nguyen Hoa ${ }^{2}$, Phawut Nueangphuet ${ }^{1}$, Ohnmar Myint ${ }^{1}$, \\ Quang Lam Truong ${ }^{3}$, Thi Lan Nguyen ${ }^{2}$, Junzo Norimine ${ }^{3}$, Takuya Hirai ${ }^{3}$, and Ryoji \\ Yamaguchi $^{1}$ \\ ${ }^{1}$ University of Miyazaki \\ ${ }^{2}$ Vietnam National University of Agriculture \\ ${ }^{3}$ Affiliation not available
}

April 27, 2020

\begin{abstract}
African swine fever (ASF) is emerging in Vietnam and poses a continuing severe threat to the swine industry. A histopathological study of clinical samples collected during the August to September 2019 outbreak of ASF was performed to determine the characteristic lesions. We analyzed samples from eight ASFV-infected farms. Histopathological results revealed the characteristic lesions of the acute to the subacute clinical form of ASF. Immunohistochemical results showed ASFV viral antigen distribution in mononuclear cells/macrophage in various organs, hepatocytes, and renal tubular epithelium. Molecular analysis of partial capsid protein 72 gene revealed that ASFV strain from the eight separate outbreaks belonged to genotype II.
\end{abstract}

\section{Title}

Pathological investigation and viral antigen distribution of emerging African Swine Fever in Vietnam

\section{Running Title}

Pathology of the emerging ASF in Vietnam

\section{Authors}

Uda Zahli Izzati ${ }^{1}$, Minori Inanaga ${ }^{1}$, Nguyen Thi Hoa ${ }^{2}$, Phawut Nueangphuet ${ }^{1}$, Ohnmar Myint ${ }^{1}$, Truong Quang Lam ${ }^{2}$, Nguyen Thi Lan ${ }^{2}$, Junzo Norimine ${ }^{1}$, Takuya Hirai ${ }^{1}$, Ryoji Yamaguchi ${ }^{1}$

\section{Affiliations}

${ }^{1}$ University of Miyazaki, Miyazaki City, Miyazaki, Japan

${ }^{2}$ Vietnam National University of Agriculture, Gia Lam, Hanoi, Vietnam

Address for correspondence: Ryoji Yamaguchi, Department of Veterinary Pathology, Faculty of Agriculture, University of Miyazaki, Gakuen-kibanadai-nishi-1-1, Miyazaki, 889-2192, Japan; email: a0d402u@cc.miyazakiu.ac.jp

\section{Summary}

African swine fever (ASF) is emerging in Vietnam and poses a continuing severe threat to the swine industry. A histopathological study of clinical samples collected during the August to September 2019 outbreak of ASF was performed to determine the characteristic lesions. We analyzed samples from eight ASFV-infected farms. 
Histopathological results revealed the characteristic lesions of the acute to the subacute clinical form of ASF. Immunohistochemical results showed ASFV viral antigen distribution in mononuclear cells/macrophage in various organs, hepatocytes, and renal tubular epithelium. Molecular analysis of partial capsid protein 72 gene revealed that ASFV strain from the eight separate outbreaks belonged to genotype II.

Keywords: Asfarviridae, domestic pig, histopathology, immunohistochemistry, natural outbreak

\section{Introduction}

The aetiologic agent for African swine fever (ASF) is the African swine fever virus (ASFV), a doublestranded DNA virus with a diameter of 70-100 $\mathrm{nm}$ and the sole member of the family Asfaviridae and the genus Asfivirus (Dixon et al, 2012). According to the first description of ASF, ASFV was transmitted from wild African suids in Kenya to domestic pigs, resulting in 100\% mortality (Montgomery, 1921). Currently, the disease is present on the continents of Africa, Europe and Asia (World Organisation for Animal Health, 2019).

The first outbreak of ASF in Vietnam was reported in February 2019 in Hung Yen province located in the northern area of Vietnam, $250 \mathrm{~km}$ from the China border (Le et al, 2019). A year after the first outbreak, ASFV was widespread within all 63 provinces in the country, with 4126 ongoing outbreaks reported by March 2020 (World Organisation for Animal Health, 2020). It has been reported that as a result of national disease control efforts, outbreaks were showing signs of slowing down by the end of 2019. However, in some provinces where the ASF epidemic was declared to be over (i.e., when there are no further ASF cases detected after a 30-day period), the ASF re-appeared (The United States Department of Agriculture, 2019).

The ASF clinical forms - peracute, acute, subacute and chronic manifestation of infected pigs-depend on the strain of the virus, route of transmission, dose and the host characteristics (Sánchez-Vizcaíno et al, 2015). The ASFV strains can also be classified as highly virulent, moderately virulent or mildly (low) virulent (Pan \& Hess 1984), and there are 24 genotypes of ASFV strains (Quembo et al, 2008). After the onset of the clinical signs of ASFV-infected pigs, the virus is shed at high levels in all secretions and excretions, and surviving pigs show long-term viremia, becoming an important ASFV reservoir in domestic pigs (Sanchez-Vizcaino et al, 2019).

ASFV is an exotic disease in the Southeast Asia region. It is a real threat to the swine industry in developing countries, especially where the majority of pig farmers are smallholders or practice backyard farming with limited biosecurity measures. As the description of histopathological lesions observed in the natural cases of ASF was limited in the literature, this study focused on the description of the histopathological lesions and viral antigen distribution in pigs naturally infected with ASFV to enhance our understanding of the pathological imaging and advance our insight onto the pathogenesis of ASFV during an ongoing outbreak.

\section{Materials and Methods}

\section{Sample collection}

During the period of ASFV outbreaks in northern Vietnam, infected pigs showed clinical signs of high fever $\left(41-42^{\circ} \mathrm{C}\right)$, diarrhea, vomiting, anorexia and reflexes, depression, dyspnea, haemorrhages in the skin, neurological signs, coma and death. Typical gross findings at the post-mortem examination were haemorrhagic lesions in multiple organs, including diffuse haemorrhage in superficial inguinal (Fig 1a), mandibular, pulmonary, gastrohepatic, renal and mesenteric (Fig 1b) lymph nodes, in addition to hyperemic splenomegaly with friable consistency and dark discoloration (Fig 1c), petechial haemorrhage in the renal cortex, diffuse haemorrhage in the renal pelvis (Fig 1d), haemorrhage in pericardium and myocardium (Fig 1e, f), swollen liver and oedema of the gall bladder wall, hemorrhagic cerebral meninges with oedema and interstitial oedema of the lungs. A blood sample was collected for real-time polymerase chain reaction (qPCR) work, and tissue samples from various organs were fixed in $10 \%$ neutral buffered formalin and routinely processed for histopathological examination. Background information for each pig was tabulated in Table 1.

Ethics statement: The diseased pigs were collected at moribund stage and humanely euthanized by vete- 
rinarians. There was no unnecessary pain inflicted on the animals because all sampling procedures were conducted during necropsy.

\section{Real-time PCR and molecular genotyping of ASFV}

DNA was extracted directly from the blood using a nucleic acid extraction kit (DNeasy Blood \& Tissue

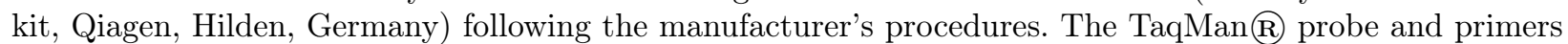
described previously were used for the detection of $250 \mathrm{bp}$ of 3'-end of the p72 gene for qPCR (King et al, 2003) for initial diagnosis. Then, $478 \mathrm{bp}$ fragments corresponding to the C-terminal end of the p72 gene were amplified for genomic sequencing using primers as described previously (Bastos et al, 2003). The PCR amplicons were purified using a QIAquick PCR Purification kit (Qiagen, Hilden, Germany). The sequence cycles were performed with the Genome Lab DTCS-Quick Start Kit (Beckman Coulter, California, USA) and analyzed by the CEQ-8000 Genetic Analysis System (Beckman Coulter, California, USA). The sequence data (456 bp) in this study and thirty partial/complete B646L gene sequences encode for major ASFV capsid p72 protein as representative from each genotype as described previously $(9)$ were retrieved from the gene bank aligned and edited to 399 nucleotides for uniformity using the BioEdit Sequence Alignment Editor (Hall, 1999). The phylogenetic analysis was conducted by the neighbor-joining approach with 1,000 bootstrap replications using MEGA X software (Saitou \& Mei, 1987; Felsenstein, 1985; Kimura, 1980; Kumar et al, 2018).

\section{Histopathology and immunohistochemistry (IHC)}

Five micrometer-thick sections of the paraffin-embedded formalin-fixed tissue samples were stained with routine hematoxylin and eosin (HE) for the histopathological evaluation. All lesions observed in the HE staining tissue specimens were examined. All tissues were tested for the ASFV antigen using the IHC method. The rabbit polyclonal ASFV phosphoprotein p30 antibody (Alpha Diagnostic International, San Antonio, Texas, USA) specific to ASFV phosphoprotein p30 was used as the primary antibody. The primary antibody was diluted at 1:2500 in Blocking One (Nacalai Tesque, Japan) solution after the optimal concentration was determined. Following heat-induced antigen retrieval in citrate buffer $\mathrm{pH} 6.0$, the primary antibody was incubated on the tissue specimen at $4{ }^{\circ} \mathrm{C}$ overnight in a humidified chamber. Histofine $\mathrm{R}$ MAX PO Multi (Nichirei Biosciences, Japan) was used as the secondary antibody, and the reaction was visualized using 3,3'-diaminobenzidine (Sigma-Aldrich) chromogen. Tissue specimens collected from a pig at an ASFV-free farm were used as negative control.

\section{Results}

\section{Real-time PCR detection and sequence analysis of ASFV}

The cycle threshold (Ct) value of ASFV detected from the blood sample and the respective name of isolate from each pig were tabulated in Table 1. Phylogenetic tree (Fig 2) based on 399 nucleotides of partial capsid p72 protein genome revealed that the ASFV strains from all eight cases in this study belonged to genotype II, 100\% similar to genotype II strains reported in Africa (AY351518, AF270706), Eurasia (KJ195685, FR682468, KM374558), China (MK33180) and Vietnam (MK554698). The sequence data of each case was submitted to the GenBank database under accession number from MT232743 to MT232750. Address is as follows: http://www.ncbi.nlm.nih.gov.

\section{Histopathology and immunohistochemical (IHC) detection of ASFV antigen}

\section{Histopathological findings}

Microscopic lesions of cases 1-8 were tabulated in Table 2. All eight cases showed haemorrhage with variable severity in the splenic tissue (Fig 3a-c). In 3/8 pigs (cases 20, 58 and 59), severe haemorrhage replaced the red pulp parenchyma and many tissues surrounding the lymphoid follicles, rendering the evaluation of necrotizing tissue difficult. In the liver tissue, the hemorrhagic lesion was seen in different levels of severity in $6 / 8$ cases. The mild cases showed focal haemorrhage in zone 2 , and severe cases showed multifocal to more extensive haemorrhages (Fig 3d-f). In the tonsillar tissue, all cases did not show any obvious significant 
changes in the tonsillar morphology, and the lymphoid follicles were easily identified in low magnification (x40, light microscope, field number 18) (Fig 4a). In higher magnification, apoptotic tissues, characterized by numerous nuclear debris and karyorrhexis, were more intense within the diffuse lymphoid tissue (T-cells area) compared to the lymphoid follicles (Fig 4b-c). Extensive necrosis or haemorrhages were not seen in all cases. In the lymph node tissue, sinus haemorrhages, apoptotic bodies and necrosis were remarkable in all cases (Fig 5a). Like the tonsil, lymphoid follicles were easily identifiable in the lymph node tissue.

In the lung tissue, interstitial pneumonia was seen in all 8 cases. Mononuclear inflammatory cell infiltration increased the thickness of the interstitium (Fig 5b). In many cases, pulmonary intravascular macrophages were easily detectable in the small blood vessels and capillaries. No prominent alveolar macrophages were seen in all cases. Pulmonary oedema was seen in $5 / 8$ cases, while pulmonary congestion $(3 / 8)$ and haemorrhage $(2 / 8)$ were uncommon. In the brain tissue, meningitis $(8 / 8)$ and perivascular cuffing $(5 / 8)$ were characterized with 2-4 layers of mononuclear cells with frequent apoptotic bodies or nuclear karyorrhexis (Fig 5c). Rarely, neutrophils were found in the meninges among the mononuclear cell infiltration. Severe congestion of the meningeal capillaries was common, but haemorrhages were subtle and not present in most of the cases. Perivascular cuffing was commonly accompanied with swollen endothelial cells. Glial nodule was absent in all cases.

In the cardiac tissue, epicardial and myocardial haemorrhage was seen in $3 / 8$ cases (Fig $5 \mathrm{~d}$ ). The haemorrhagic areas were sometimes infiltrated with aggregates of mononuclear inflammatory cells. Microscopically, haemorrhagic lesions were seen in various locations of the gastrointestinal tract tissue. Case 58 showed haemorrhage in the lamina propria of gastric tissue with oedema in the submucosal layer (Fig 5e). Case 18 showed multifocal haemorrhages in the outer muscularis layer of the small intestinal tissue (Fig $5 \mathrm{f}$ ). Case 19 showed haemorrhages in the deep lamina propria, dilated capillaries with congestion, and oedema in the submucosa layer of large intestinal tissue (Fig $5 \mathrm{~g}$ ). In the renal tissue, all cases showed haemorrhage in the renal pelvis (Fig 5h), but only cases 57 and 59 showed oedemas in the area. Petechial haemorrhages of the renal cortex were seen in 5/8 cases, with the lesion occasionally extended into the capsular surface (Fig 5i). The haemorrhagic lesion was absent in the renal medulla. Multifocal interstitial nephritis was seen in $5 / 8$ cases.

\section{ASFV antigen detection by immunohistochemistry (IHC)}

ASFV antigen-labelled cells were detected in various organs and majorly found in the mononuclear cells/macrophages. The result was tabulated in Table 3 . The labelled cells were commonly detected in the lymphoid organs (spleen, tonsil and lymph node) as well as lung, kidney and liver tissues. Figure 6a showed the positive cells in the background of the haemorrhagic spleen. In the liver, positive cells were found in the sinusoids, and they were mostly enlarged, plump Kupffer cells (Fig 6b). In a severe case, ASFV antigen-labelled hepatocytes were found (Fig 6c). In the tonsil, positive mononuclear cells were seen in the epithelium of the tonsillar crypt (M-cell) and mostly within the diffuse lymphoid area surrounding the lymphoid follicles (Fig 6d). In the lymph node, positive cells were numerous in some cases and difficult for numeration because the cells lost their distinctive shape and blended with the background. Figure 6e showed the ASFV antigen-positive macrophages with many of them containing phagocytosed erythrocytes. In the lungs, pulmonary intravascular macrophages were commonly found positive (Fig 6f). In the cerebrum, positive cells were scarcely found. Figures $6 \mathrm{~g}$ and $6 \mathrm{~h}$ showed a few positive mononuclear cells in meningitis and perivascular cuffing lesions. In the epicardium of the heart, other than mononuclear cells/macrophages, many spindle-shaped mononuclear cells were positive in the haemorrhagic area (Fig 6i). In the kidney tissue, many spindle-shaped cells were stained positive in addition to plump-shaped macrophages within the haemorrhagic renal pelvis region (6j). Occasionally, a few positive cells were found in the capillary of glomeruli identified as macrophages (Fig 6k). Multifocally, the renal tubules of the kidneys showed granular cytoplasmic staining of ASFV antigen (Fig 6l). Tissue sections from negative control pig showed no ASFV antigen-labelled cells or non-specific staining in all tissues.

\section{Discussion}


The genotype II of ASFV strains in this study showed acute to subacute forms of ASFV based on the clinical presentation as well as gross and histopathological findings. A $70 \%$ mortality rate was estimated in a single farm (case 74), whilst the accurate mortality rates in seven other affected farms were undetermined because of the rapid enforcement of herd depopulation. The main lesions of acute ASF, including hyperemic splenomegaly, haemorrhagic lymph nodes, especially in the peripheral lymphatic sinus, petechial haemorrhage in the renal cortex, pulmonary oedema, haemorrhages in the epicardium, and subacute ASF, including hyperemic splenomegaly, severe and more diffuse haemorrhagic lymph nodes (cortical and medulla), petechial haemorrhages in the cortex and pelvis, gall bladder wall oedema, haemorrhages in the epicardium, as reported previously (Sánchez-Vizcaíno et al, 2015), were seen among the eight cases studied.

There are several known viral pathogens causing porcine haemorrhagic disease in Vietnam, including highly pathogenic porcine reproductive and respiratory syndrome virus (HP-PRRSV) and classical swine fever virus (CSFV), which produced very similar haemorrhagic lesions in the lymph nodes (Huong Giang et al, 2016; Choe et al, 2020). Antigen detection of HP-PRRSV and CSFV by the RT-PCR method yielded negative results (data not shown), confirming that the lesions seen in those tissues were highly attributable to ASFV infection. Even though ASFV mainly targeted the macrophages/mononuclear phagocytic system similar to both HP-PRRSV and CSFV, the histopathological lesions in the lymph node tissues were found to be different among the viruses. There were marked histiocytic hyperplasia in CSFV infection, which is not seen in ASFV infection. In contrast, CSFV infection manifested more marked and severe lymphoid depletion (disappearance of lymphoid follicles) compared to HP-PRRSV infection in relation to the strain causing outbreaks in Vietnam from 2010 to 2018 (personal observation). Bacterial diseases causing similar haemorrhagic lesions, such as in septicemic salmonellosis and swine erysipelas, were also ruled out based on negative PCR detection (data not shown) and the lack of histopathological characteristics of both bacterial infections.

ASFV primary replication site is in the mononuclear cells/macrophages; therefore, the ASFV antigen was consistently detected in a large number in the lymphoid organs. In the lymph node, ASFV causes apoptosis of lymphocytes, mainly in the T cells compared to B cells (Carrasco et al, 1996). The death of the lymphocytes is caused by an indirect mechanism involving the release of proinflammatory cytokines, mainly tumor necrosis factor- $\alpha$, interleukin- 1 and interleukin- 6 by activated monocytes and macrophages (Salguero et al, 2005), as the virus had been shown not to replicate in the lymphocytes (Carrasco et al, 1996). Similarly, we observed apoptotic bodies more intensely distributed in the diffuse lymphoid tissue ( $\mathrm{T}$ cell area) of tonsil and lymph nodes in this study. IHC also showed frequent ASFV antigen-labelled mononuclear cells/macrophages in the diffuse lymphoid tissue area compared to the lymphoid follicles. In the spleen, the degree of haemorrhage was very severe in several cases, resulting in very few viable cells surrounding the follicular structures and severe lymphoid depletion. This phenomenon was described as a consequence of necrotic fixed macrophages of the splenic cords and subsequent deposition of fibrin (Carrasco et al, 1997). However, we did not observe frequent fibrin deposition in all the cases, including in the most severely haemorrhagic splenic tissue.

During the outbreak, ASFV-infected pigs showed neurological signs of paddling, spasm and convulsion in lateral recumbency before the loss of consciousness, coma or death. In the central nervous system, histopathological lesion attributed to ASFV was perivascular cuffing in the brain, which contained more nuclear debris than what is seen in CSFV infection (Robinson \& Robinson, 2016). The detection of ASFV antigen by IHC in the brain tissue of a domestic pig is rarely reported elsewhere. In this study, the ASFV antigen was presented in the mononuclear cells of the meninges (with or without clear haemorrhage) and perivascular cuffing in the grey matter of the cerebral cortex, which may contribute to the characteristic lesions seen in the brain tissue. Notably, the number of ASFV antigen-labelled cells was significantly fewer compared to other tissues in this study, and a single case of non-suppurative meningoencephalitis showed negative ASFV antigen. These findings may support the role of proinflammatory cytokines contributing to the inflammatory lesion in the brain.

In the lungs, pulmonary intravascular macrophages (PIMs) were easily identified in the ASFV-infected pigs. Based on the IHC results, lung tissue is also a primary site of ASFV antigen detection. PIMs play a significant 
role in several species of animals, including ruminants, cats, pigs and horses as the primary cells in defense mechanisms against hematogenous pathogens (López \& Martinson, 2017), and increases in the size, number and phagocytic activity of PIMs were reported during an ASF viral infection (Carrasco et al, 2002). In acute ASF cases, the release of proinflammatory cytokines as mentioned from activated PIMs lead to increased vascular permeability, resulting in pulmonary oedema (Carrasco et al, 1996). However, there were no obvious correlations between the number of ASFV antigens found in the lung tissue and the occurrence of pulmonary oedema observed in this study.

In the liver, multifocal necrosis and hemadsorption phenomenon were reported in Kupffer cells, circulating monocytes and hepatocytes (Fernandez et al, 1992; Sierra et al, 1987). In this study, ASFV caused multifocal haemorrhages mainly in the midzonal (zone 2) region of the hepatic lobules with different severity of necrosis. Numerous Kupffer cells/circulating macrophages in the sinusoids and haemorrhagic areas were labelled with ASFV antigen, but positively-labelled hepatocytes were rare, except in a severe case (case 20). In severe cases, more generalized, coalescing multifocal haemorrhages produce an appearance of selective midzonal (zone 2) to nearly centrilobular necrosis with haemorrhage. The lesion observed in this study showed the midzonal necrosis was frequently accompanied by haemorrhages and mononuclear cell infiltrations, while most of the hepatocytes in zones 1 and 3 remained less affected. Only one case showed a negative detection of ASFV despite the presence of histopathological lesions, which may require further investigation.

Multifocal haemorrhages were found in the heart, gastrointestinal tract and kidney tissues. There was a presence of many spindle-shaped cells labelled with ASFV antigen in the haemorrhagic lesion of the epicardium and renal pelvis, which were not identified as macrophages by IHC using Iba-1 antibody (data not shown). The origin of these cells was not confirmed; however, their morphology may suggest a fibroblastic origin. The significance of these ASFV infected-cells contributing to the lesion in the epicardium and renal pelvis is not known, but infected fibroblasts and smooth muscle cells in the later stage of infection have been reported (Blome et al, 2013). Multifocal haemorrhages in the gastrointestinal tracts vary in their location, localized in the lamina propria of gastric and large intestinal epithelium or only presented in the outer muscularis layer of the small intestine. There are few ASFV antigen-labelled cells found in these areas, which may imply the lesions mainly attributed to the concentration of cytokines reaching and affecting the area by the blood circulation. In ASFV-infected pigs, the gastrohepatic, renal and mesenteric lymph nodes were some of the major lymph nodes with haemorrhagic lesions. The anatomical location of the gastrointestinal tract in the vicinity of affected lymph nodes may have contributed to the large concentration of proinflammatory cytokines supplied to those organs by the blood or lymph, causing the vascular-related changes in those organs. The factors relating to such occurrence require further pathological investigation.

Reportedly, renal tubular epithelium is not a leading replication site of ASFV. Among the eight cases studied, three cases (case 58,59,60) showed the absence of renal epithelium labelled with an ASFV antigen. Furthermore, the staining pattern of the ASFV-labelled renal epithelium showed a granular cytoplasmic pattern, instead of diffuse, intense staining in the cytoplasm of macrophage/mononuclear cells. Instead of an active viral replication site, it may be inferred that there was a high load of ASF viral particle reabsorption from the urine in the renal tubular epithelium.

In the lymphoid tissues, the ASFV positive cells were consistently observed with no regard for the severity in the apoptotic, haemorrhage and necrotic lesions in those tissues. However, in many other tissues of different organs, there were instances where the number of ASFV antigen was few or negative even though haemorrhagic lesions, as discussed previously, were observed. On the other hand, there were also instances where the ASFV antigen was observed without obvious lesions. The amount of proinflammatory cytokines produced by the infected cells in the main target organs (lymphoid organs) may have a greater influence on the production of the pathological lesions at distant organs rather than the number of infected cells presented in those organs.

In the absence of an effective treatment or vaccination against ASFV, the depopulation of infected pig herd and all herds surrounding the affected farm in a high-density pig area resulted in a significant monetary loss to the Vietnam's majority smallholder and backyard farmer. In ASF-endemic countries, it is worth noting 
that the ASFV may become less virulent and infected pigs may show less severe clinical presentation in which case the histopathological lesions may eventually vary in the future (Robinson \& Robinson, 2016). Thus, this study highlighted the histopathological findings of ASFV genotype II in its initial epidemic in Vietnam.

\section{Acknowledgements}

We thank all veterinarians and personnel involved in conducting the necropsy of diseased pigs during the outbreak.

\section{Conflict of interest statement}

The authors declare that they have no conflicts of interest.

\section{Funding}

This work was supported by Japan Society for the Promotion of Science (JSPS) KAKENHI Grant Number JP17H04639.

\section{References}

1. Bastos, A. D. S., Penrith, M.-L., Crucière, C., Edrich, J. L., Hutchings, G., Roger, F., Couacy-Hymann, E., \& R.Thomson, G. (2003). Genotyping field strains of African swine fever virus by partial p72 gene characterisation. Archives of Virology, 148, 693-706. https://doi.org/10.1007/s00705-002-0946-8

2. Blome, S., Gabriel, C., \& Beer, M. (2013). Pathogenesis of African swine fever in domestic pigs and European wild boar. Virus Research, 173(1), 122-130. https://doi.org/10.1016/j.virusres.2012.10.026

3. Carrasco, L., Bautista, M., Gómez-Villamandos, J., Martin de las Mulas, J., Chacón-M de lara, Wilkinson, P. J., \& Sierra, M. (1997). Development of microscopic lesions in splenic cords of pigs infected with African swine fever virus. Veterinary Research, 28, 93-99. PMID: 9172845

4. Carrasco, L., Chacón-m de Lara, F., Gómez-Villamandos, J. C., Bautista, M. J., Villeda, C. J., Wilkinson, P. J., \& Sierra, M. A. (1996). The pathogenic role of pulmonary intravascular macrophages in acute African swine fever. Research in Veterinary Science, 61(3), 193-198. https://doi.org/10.1016/S00345288(96)90062-4

5. Carrasco, L., Chacón-M. de Lara, F., Martín de las Mulas, J., Gómez-Villamandos, J. C., Pérez, J., Wilkinson, P. J., \& Sierra, M. A. (1996). Apoptosis in lymph nodes in acute African swine fever. Journal of Comparative Pathology, 115(4), 415-428. https://doi.org/10.1016/S0021-9975(96)80075-2

6. Carrasco, L., Núñez, A., Salguero, F. J., Diaz San Segundo, F., Sánchez-Cordón, P., Gómez-Villamandos, J. C., \& Sierra, M. A. (2002). African Swine Fever: Expression of Interleukin-1 alpha and Tumour Necrosis Factor-alpha by Pulmonary Intravascular Macrophages. Journal of Comparative Pathology, 126(2-3), 194-201. https://doi.org/10.1053/jcpa.2001.0543

7. Choe, S., Le, V. P., Shin, J., Kim, J.-H., Kim, K.-S., Song, S., Cha, R. M., Park, G.-N., Nguyen, T. L., Hyun, B.-H., Park, B.-K., \& An, D.-J. (2020). Pathogenicity and Genetic Characterization of Vietnamese Classical Swine Fever Virus: 2014-2018. Pathogens, 9(3), 169. https://doi.org/10.3390/pathogens9030169

8. Dixon, L. K., Alonso, C., Escribano, J. M., Martins, C., Revilla, Y., Salas, M. L., et al. (2012). Family Asfarviridae. In: Virus Taxonomy. Classification and Nomenclature of Viruses. Ninth Report of the International Committee on Taxonomy of Viruses. King, A. M. Q., Adams, M. J., Carstens, E. B., Lefkowitz, E. J., Ed. Elsevier. pg 153-162.

9. Felsenstein, J. (1985). Confidence limits on phylogenies: An approach using the bootstrap. Evolution, 39, 783-791. https://doi.org/10.1111/j.1558-5646.1985.tb00420.x

10. Fernandez, A., Perez, J., Martin de las Mulas, J., Carrasco, L., Dominguez, J., \& Sierra, M. (1992). Localization of African Swine Fever viral antigen, swine IgM, IgG and C1q in lung and liver tissues of experimentally infected pigs. J. Comp. Path., 107, 81-90. https://doi.org/10.1016/0021-9975(92)90098$\mathrm{F}$

11. Hall, T. A. (1999). BioEdit: a user-friendly biological sequence alignment editor and analysis program for Windows 95/98/NT. Nucleic Acids Symposium Series, 41, 95-98. 
12. Huong Giang, N. T., Lan, N. T., Nam, N. H., Hirai, T., \& Yamaguchi, R. (2016). Pathological Characterization of an Outbreak of Porcine Reproductive and Respiratory Syndrome in Northern Vietnam. Journal of Comparative Pathology, 154, 135-149. https://doi.org/10.1016/j.jcpa.2015.11.004

13. Kimura, M. (1980). A simple method for estimating evolutionary rate of base substitutions through comparative studies of nucleotide sequences. Journal of Molecular Evolution, 16, 111-120. https://doi.org/10.1007/BF01'

14. King, D. P., Reid, S. M., Hutchings, G. H., Grierson, S. S., Wilkinson, P. J., Dixon, L. K., Bastos, A. D. S., \& Drew, T. W. (2003). Development of a TaqManß PCR assay with internal amplification control for the detection of African swine fever virus. Journal of Virological Methods, 107, 53-61. https://doi.org/10.1016/S0166-0934(02)00189-1

15. Kumar, S., Stecher, G., Li, M., Knyaz, C., \& Tamura, K. (2018). MEGA X: Molecular Evolutionary Genetics Analysis across computing platforms. Molecular Biology and Evolution, 35, 1547-1549. https://doi.org/10.1093/molbev/msy096

16. Le, V. P., Jeong, D. G., Yoon, S.-W., Kwon, H.-M., Trinh, T. B. N., Nguyen, T. L., Bui, T. T. N., Oh, J., Kim, J. B., Cheong, K. M., Van Tuyen, N., Bae, E., Vu, T. T. H., Yeom, M., Na, W., \& Song, D. (2019). Outbreak of African Swine Fever, Vietnam, 2019. Emerging Infectious Diseases, 25, 1433-1435. https://doi.org/10.3201/eid2507.190303

17. López, A., Martinson, S. A. (2017). Respiratory System, Mediastinum, and Pleurae . In: Pathologic basis of veterinary diseases. Zachary, J. F., Ed. Elsevier. pg 483.

18. Montgomery, R. E. (1921). On A Form of Swine Fever Occurring in British East Africa (Kenya Colony). Journal of Comparative Pathology and Therapeutics, 34, 159-191. https://doi.org/10.1016/S03681742(21)80031-4

19. Pan, I. C., Hess, W. R. (1984). Virulence in African Swine Fever-Its measurement and implications. American Journal of Veterinary Research, 45, 361-366. PMID: 6711963

20. Quembo, C. J., Jori, F., Vosloo, W., \& Heath, L. (2018). Genetic characterization of African swine fever virus isolates from soft ticks at the wildlife/domestic interface in Mozambique and identification of a novel genotype. Transboundary and Emerging Diseases, 65, 420-431. https://doi.org/10.1111/tbed.12700

21. Robinson, W. F., Robinson, N. A. (2016). Cardiovascular System . In: Jubb, Kennedy, and Palmer's Pathology of Domestic Animals, Vol 3, $6^{\text {th }}$ Edit. Maxie, M. G., Ed. Elsevier. pg 74-76.

22. Saitou, N., \& Nei M. (1987). The neighbor-joining method: A new method for reconstructing phylogenetic trees. Molecular Biology and Evolution, 4, 406-425. https://doi.org/10.1093/oxfordjournals.molbev.a040454

23. Salguero, F. J., Sánchez-Cordón, P. J., Núñez, A., Fernández de Marco, M., \& Gómez-Villamandos, J. C. (2005). Proinflammatory Cytokines Induce Lymphocyte Apoptosis in Acute African Swine Fever Infection. Journal of Comparative Pathology, 132(4), 289-302. https://doi.org/10.1016/j.jcpa.2004.11.004

24. Sánchez-Vizcaíno, J. M., Mur, L., Gomez-Villamandos, J. C., \& Carrasco, L. (2015). An Update on the Epidemiology and Pathology of African Swine Fever. Journal of Comparative Pathology, 152, 9-21. https://doi.org/10.1016/j.jcpa.2014.09.003

25. Sanchez-Vizcaino, J., Laddomada, A., Arias, M. L. (2019).African Swine Fever Virus. In: Diseases of Swine. Zimmerman, J. J., Karriker, L. A., Ramirez, A., Schwartz, K. J., Stevenson, G. W., Zhang, J., Ed. John Wiley \& Sons. pg 443-452.

26. Sierra, M. A., Bernabe, A., Mozos, E., Mendez, A., \& Jover, A. (1987). Ultrastructure of the Liver in Pigs with Experimental African Swine Fever. Veterinary Pathology, 24(5), 460-462. https://doi.org/10.1177/0300985887

27. The United States Department of Agriculture. 2019. Vietnam African Swine Fever Update, December 06, 2019. Retrieved from: https://apps.fas.usda.gov/newgainapi/api/Report/DownloadReportByFileName?fileName=V 30-2019 [cited 2020 March 10].

28. World Organisation for Animal Health. 2019. African Swine Fever (ASF) Report N¹7: 2016 - 2019. Retrieved from https://www.oie.int/fileadmin/Home/eng/Animal_Health_in_the_World/docs/pdf/Disease_cards/ASF/Re] [cited 2020 March 17].

29. World Organisation for Animal Health. 2020. African Swine Fever (ASF) Report N 39: February 28 to March 12, 2020. Retrieved from https://www.oie.int/fileadmin/Home/eng/Animal_Health_in_the_World/docs/pdf/Dise [cited 2020 March 19].

Table 1. Information of the pigs in the African swine fever outbreak in August-September 2019, Vietnam 


\begin{tabular}{|c|c|c|c|c|c|c|}
\hline Case ID & $\mathrm{Age}^{+}$ & Province & Farm's $\mathrm{SPP}^{++}$ & Clinical signs & $\begin{array}{l}\text { qPCR Ct } \\
\text { value }^{\S}\end{array}$ & $\begin{array}{l}\text { ASFV isolate } \\
\text { name }\end{array}$ \\
\hline 18 & 13 & Hung Yen & 1000 & $\begin{array}{l}\text { fever, } \\
\text { anorexia, } \\
\text { vomiting, } \\
\text { respiratory } \\
\text { distress, } \\
\text { haemor- } \\
\text { rhages in } \\
\text { the skin, } \\
\text { neurological } \\
\text { signs, coma }\end{array}$ & 13.91 & VN/Pig/HY01/2 \\
\hline 19 & 16 & Hung Yen & 500 & $\begin{array}{l}\text { fever, } \\
\text { anorexia, } \\
\text { diarrhea, } \\
\text { respiratory } \\
\text { distress, } \\
\text { haemor- } \\
\text { rhages in the } \\
\text { skin, coma }\end{array}$ & 16.27 & VN/Pig/HY02/2 \\
\hline 20 & 11 & Hung Yen & 400 & $\begin{array}{l}\text { fever, } \\
\text { anorexia, } \\
\text { respiratory } \\
\text { distress, } \\
\text { haemor- } \\
\text { rhages in } \\
\text { the skin, } \\
\text { neurological } \\
\text { signs }\end{array}$ & 15.32 & VN/Pig/HY03/2 \\
\hline 57 & 12 & Ha Nam & 800 & $\begin{array}{l}\text { fever, } \\
\text { anorexia, } \\
\text { diarrhea, } \\
\text { respiratory } \\
\text { distress, } \\
\text { neurological } \\
\text { signs }\end{array}$ & 14.72 & VN/Pig/HN04/2 \\
\hline 58 & 15 & Ha Nam & 500 & $\begin{array}{l}\text { fever, } \\
\text { anorexia, } \\
\text { diarrhea, } \\
\text { haemor- } \\
\text { rhages in } \\
\text { the skin, } \\
\text { neurological } \\
\text { signs }\end{array}$ & 14.55 & VN/Pig/HN05/2 \\
\hline
\end{tabular}




\begin{tabular}{|c|c|c|c|c|c|c|}
\hline Case ID & $\mathrm{Age}^{+}$ & Province & Farm's $\mathrm{SPP}^{++}$ & Clinical signs & $\begin{array}{l}\text { qPCR Ct } \\
\text { value }^{\S}\end{array}$ & $\begin{array}{l}\text { ASFV isolate } \\
\text { name }\end{array}$ \\
\hline 59 & 10 & Ha Nam & 250 & $\begin{array}{l}\text { fever, } \\
\text { anorexia, } \\
\text { loss of } \\
\text { appetite and } \\
\text { reflexes, } \\
\text { respiratory } \\
\text { distress, } \\
\text { coma }\end{array}$ & 15.51 & VN/Pig/HN06/2 \\
\hline 60 & 15 & Thai Binh & 300 & $\begin{array}{l}\text { fever, } \\
\text { anorexia, } \\
\text { loss of } \\
\text { appetite and } \\
\text { reflexes, } \\
\text { respiratory } \\
\text { distress, } \\
\text { haemor- } \\
\text { rhages in the } \\
\text { skin, coma }\end{array}$ & 16.59 & VN/Pig/TB07/2 \\
\hline 74 & 15 & Hung Yen & 200 & $\begin{array}{l}\text { fever, } \\
\text { anorexia, } \\
\text { vomiting, } \\
\text { diarrhea, } \\
\text { haemor- } \\
\text { rhages in } \\
\text { the skin }\end{array}$ & 17.07 & VN/Pig/HY08/2 \\
\hline
\end{tabular}

+ Week-old; ${ }^{++}$Standing pig population; ${ }^{\S} \mathrm{Ct}$ value of real-time PCR detection of African swine fever virus (cut off $=40)$

Table 2. Histopathological lesions in the African swine fever infected pigs from the outbreak in AugustSeptember 2019, Vietnam. ${ }^{+}$

\begin{tabular}{|c|c|c|c|c|c|c|c|}
\hline Organs & Histopathological findings & Case ID & Case ID & Case ID & Case ID & Case ID & Case ID \\
\hline & & 18 & 19 & 20 & 57 & 58 & 59 \\
\hline \multirow[t]{3}{*}{ Spleen } & Apoptosis & - & +++ & +++ & +++ & + & + \\
\hline & Haemorrhage & + & ++ & +++ & +++ & +++ & +++ \\
\hline & Necrosis & - & + & nil & +++ & nil & nil \\
\hline \multirow[t]{2}{*}{ Liver } & Haemorrhage & ++ & ++ & +++ & +++ & + & ++ \\
\hline & Necrosis & - & + & +++ & +++ & - & ++ \\
\hline Tonsil & Apoptosis & + & +++ & + & + & +++ & + \\
\hline \multirow[t]{3}{*}{ Lymph node } & Apoptosis & + & +++ & ++ & +++ & +++ & ++ \\
\hline & Haemorrhage & +++ & ++ & +++ & +++ & +++ & ++ \\
\hline & Necrosis & ++ & ++ & ++ & +++ & +++ & ++ \\
\hline \multirow[t]{4}{*}{ Lung } & Interstitial pneumonia & ++ & ++ & + & ++ & +++ & +++ \\
\hline & Pulmonary oedema & - & +++ & + & +++ & - & +++ \\
\hline & Haemorrhage & - & - & + & - & - & - \\
\hline & Congestion & - & ++ & - & - & + & + \\
\hline Brain & Perivascular cuffing & - & ++ & +++ & ++ & - & - \\
\hline
\end{tabular}




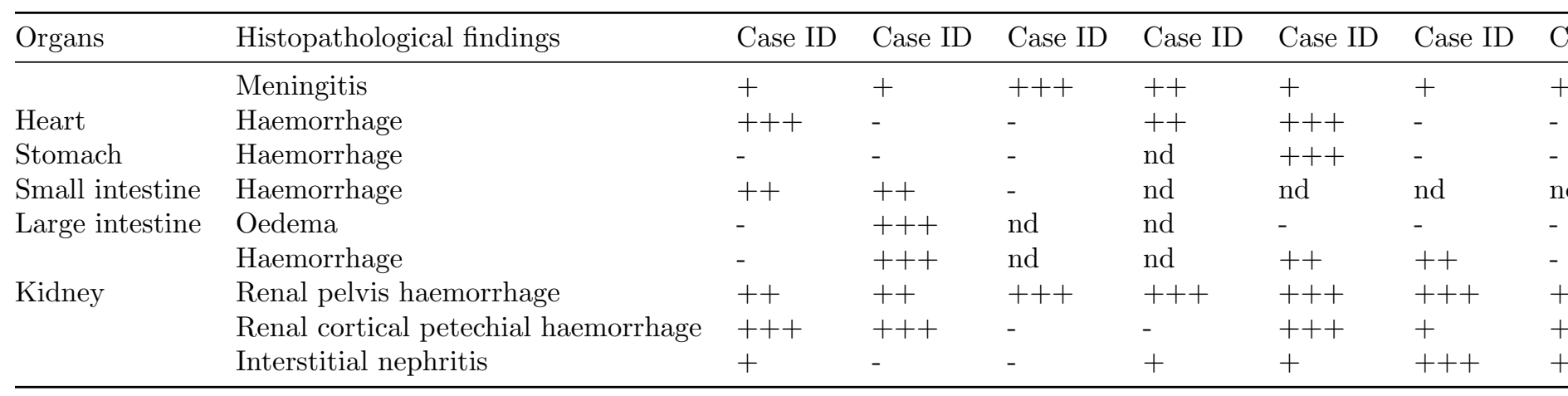

${ }_{+}^{+}$: no lesion; + : mild; ++ : moderate; +++ : severe; nil: necrotic cells not evaluated because of severe haemorrhage; nd: not done;

Table 3. African swine fever virus antigen detection by immunohistochemistry method in the infected pigs from the outbreak in August-September 2019, Vietnam. ${ }^{+}$

\begin{tabular}{|c|c|c|c|c|c|c|c|c|}
\hline Organ & Case ID & Case ID & Case ID & Case ID & Case ID & Case ID & Case ID & Case ID \\
\hline & 18 & 19 & 20 & 57 & 58 & 59 & 60 & 74 \\
\hline Spleen & ++ & +++ & +++ & ++++ & ++ & + & +++ & ++ \\
\hline Liver & ++ & ++++ & ++++ & ++ & + & 0 & +++ & ++ \\
\hline Tonsil & ++ & ++++ & +++++ & ++ & ++++ & +++ & ++ & +++++ \\
\hline Lymph node & + & +++ & +++ & +++++ & +++++ & +++ & + & +++ \\
\hline Lung & ++ & +++ & +++ & ++ & ++ & +++++ & + & ++++ \\
\hline Brain & 0 & + & + & + & 0 & + & 0 & + \\
\hline Heart & ++ & 0 & + & 0 & +++ & 0 & 0 & + \\
\hline Stomach & + & 0 & + & nd & + & + & 0 & + \\
\hline Small intestine & + & + & ++ & nd & nd & nd & nd & nd \\
\hline Large intestine & + & 0 & nd & nd & + & + & 0 & + \\
\hline Kidney & $++/$ & +1 & $+++/$ & $+/$ & $+++/$ & $++/$ & ++1 & +1 \\
\hline
\end{tabular}

+ Number of positive staining in mononuclear cell/macrophages counted in 3 fields under $\mathrm{x} 400$ magnifications (light microscope, field number 18); 0: no positive cells; +: 1-10 positive cells; ++ : 11-20 positive cells; +++ : 21-30 positive cells; ++++ : $31-40$ positive cells; $+++++:>41$ positive cells; hepatocytes and spindle-shaped cells with positive staining were not counted; nd: not done; pos: intracytoplasmic granular staining in the renal tubular epithelium; neg: no staining in the renal tubular epithelium.

\section{Figure legend}

Figure 1. Gross lesions in ASFV-infected pigs in Vietnam, 2019 (1a-f). Marble-like haemorrhage in the superficial inguinal lymph nodes, case 18 (a). Diffuse haemorrhage in the mesenteric lymph nodes, case 74 (b). Petechial haemorrhage on the renal capsule, renal cortex and diffuse haemorrhage in the renal pelvis, case 18 (c). Hyperemic splenomegaly in relation to the size of the stomach. Gastrohepatic lymph nodes showed diffuse haemorrhage, case 59 (d). Ecchymoses of the epicardium with serosanguinous pericardial fluid, case 58 (e). Ecchymoses of the myocardium, case 58 (f).

Figure 2. African swine fever virus isolates phylogenetic tree based on partial length of B646L gene encodes for the major capsid p72 protein (399 nucleotides). The black circle denotes the eight ASF cases in the August-September 2019 outbreaks in Vietnam. The phylogenetic tree was built using the neighbor-joining method with 1000 replicates bootstrap tests. 
Figure 3. Splenic tissue of an ASF-infected pig, HE staining (1a-c). Mild splenic haemorrhage in case 18. The cells of red pulp are discernible, and a few lymphoid follicles are present (a). Moderate splenic haemorrhage in case 19. There are moderate increases of erythrocytes in the red pulp and fewer discernible lymphoid follicles (b). Severe splenic haemorrhage in case 57. The red pulp parenchyma is largely replaced by erythrocytes; there are noticeable necrotic tissues and less discernable lymphoid follicles (c). Hepatic tissue of ASF-infected pig, HE staining (1d-f). Mild hepatic haemorrhage of case 58. There is a focus of haemorrhage (arrowhead) in zone 2 (d). Moderate haemorrhage in case 18. There are multifocal haemorrhages in several hepatic lobules (e). Severe haemorrhage in pig 20. There are generalized, diffuse, necrotizing haemorrhage in the hepatic parenchyma (f).

Figure 4. Tonsillar tissue of ASF-infected pig, HE staining (2a-c). The tonsil of case 20 under low magnification does not show remarkable changes (a). In higher magnification, connective fibrous tissue shows enhanced appearance (b). Apoptotic cells in the diffuse lymphoid area are easily appreciated in this magnification (asterisk). The lymphoid follicle (F) contains very few apoptotic bodies (c).

Figure 5. HE staining of tissues from ASF-infected pigs (3a-i). There is sinus haemorrhage in the lymph node tissue of case 58 (a). Interstitial pneumonia and an increased number of pulmonary intravascular macrophages are present in the lung tissue of case 58 (b). Three to four layers of mononuclear cell infiltrations in the meninges and perivascular cuffing are present in the cerebral tissue of case 20 . The inset shows swollen endothelial cells and apoptotic bodies in the perivascular cuffing lesion (c). Epicardial and myocardial haemorrhage with infiltration of mononuclear cells is present in the heart tissue of case 18 (d). Multifocal haemorrhages are seen in the superficial and deep lamina propria in the gastric epithelial tissue of case 58 (e). Multifocal haemorrhages are present in the outer longitudinal muscularis layer in the small intestinal tissue of case 18 (f). The large intestinal tissue of case 58 shows multifocal haemorrhages in the deep lamina propria and dilated capillaries filled with erythrocytes in the submucosa layer with extensive oedema beneath $(\mathrm{g})$. There is extensive haemorrhage in the pelvis region of the renal tissue in case $20(\mathrm{~h})$. Multifocal haemorrhages are found in the cortical region, sometimes extended into the capsular layer of the renal tissue in case 19 (i).

Figure 6. Immunohistochemical staining detection of ASFV antigen in the pig tissues (a-1). Most of the ASFV-positive cells are detected in necrotizing macrophages in the splenic tissue of case 57 (a). Many Kupffer cells and/or circulating macrophages are positive in the hepatic sinusoids of the liver tissue in case 20 (b). Arrowheads show hepatocytes with positive staining in the liver tissue of case 20 (c). M-cells in the epithelial crypt of tonsil and mononuclear cells in the diffuse lymphoid area are positive in the tonsillar tissue of case 20 (d). Positive cells are present in the sinus of the lymph node tissue of case 58. Many of the positive cells are erythrocyte-laden macrophages (e). Two pulmonary intravascular macrophages are positive in the lung tissue of case 58 (f). A few mononuclear cells in the cerebral meninges are positive in the cerebral tissue of case 20 (g). Positive cells are present in the perivascular cuffing in the cerebral tissue of case 20 (h). Many spindle-shaped cells are positive in the epicardial haemorrhage in the cardiac tissue of case 18 (i). Within the hemorrhagic region of the renal pelvis, macrophage/mononuclear cells and many spindle-shaped cells are positive in the renal tissue of case 20 (j). Positive macrophage/mononuclear cells are present in the capillary of a glomerulus in the renal tissue of case $18(\mathrm{k})$. Renal tubular epithelium shows positive staining in the renal tissue of case 57 (1). 


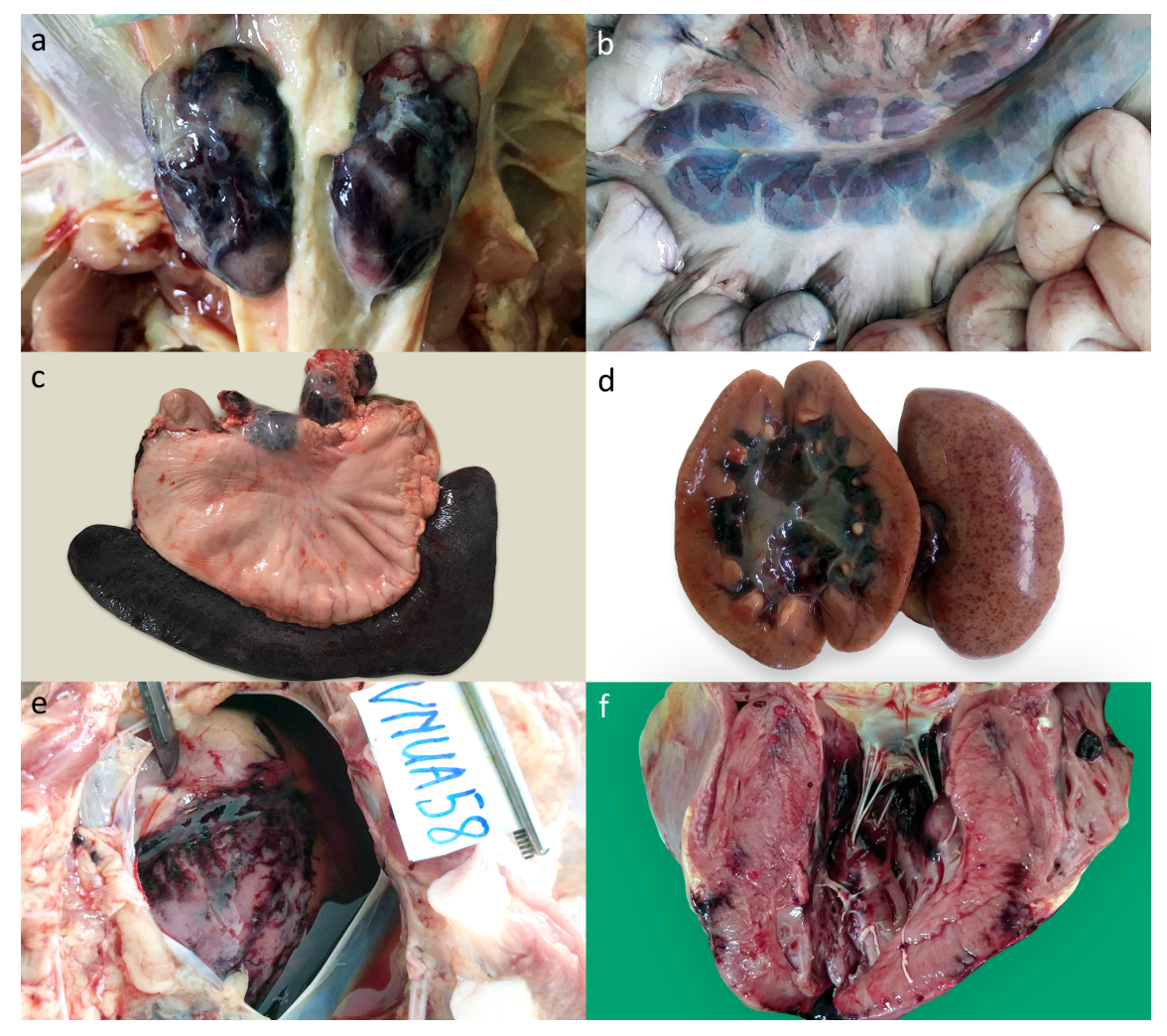




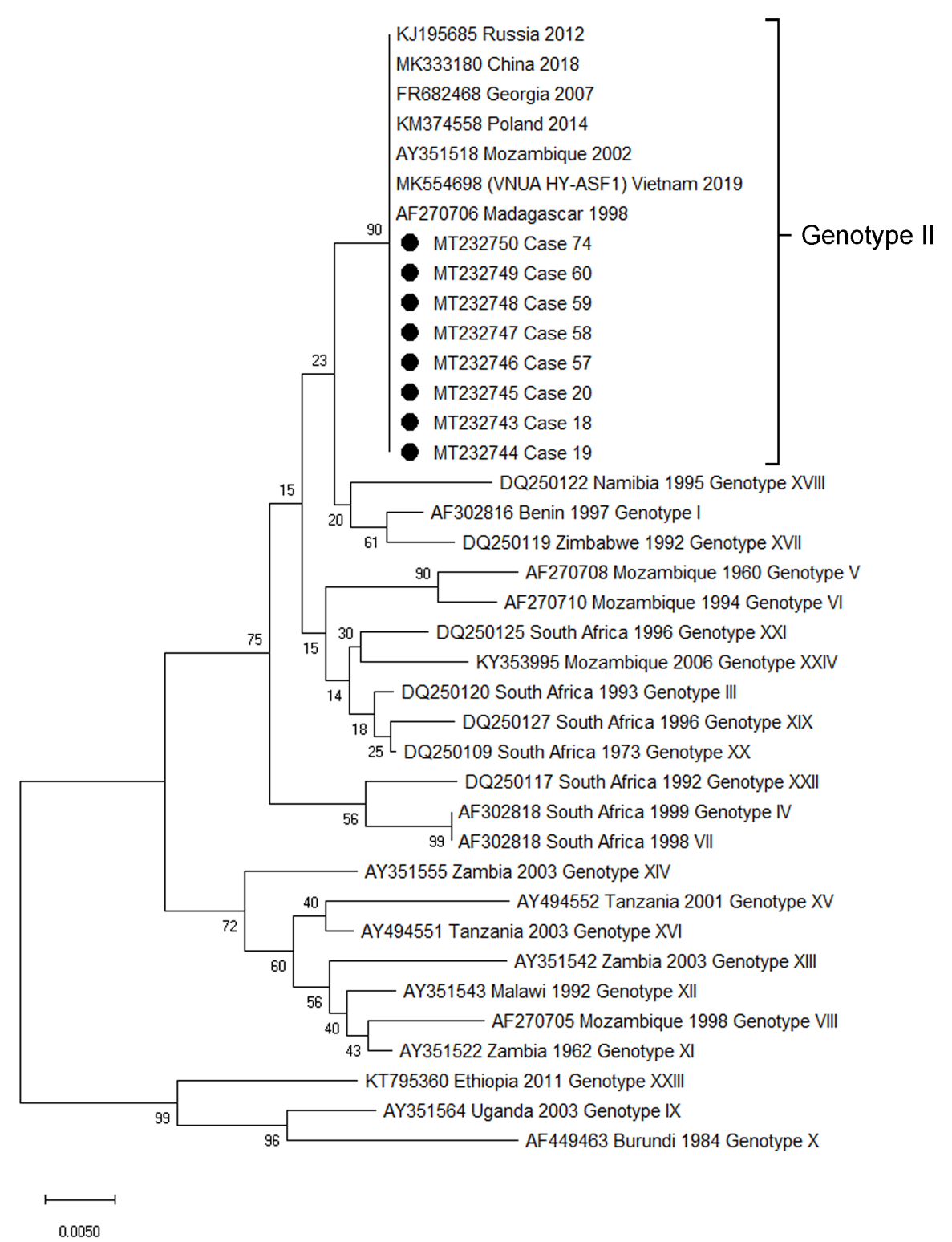



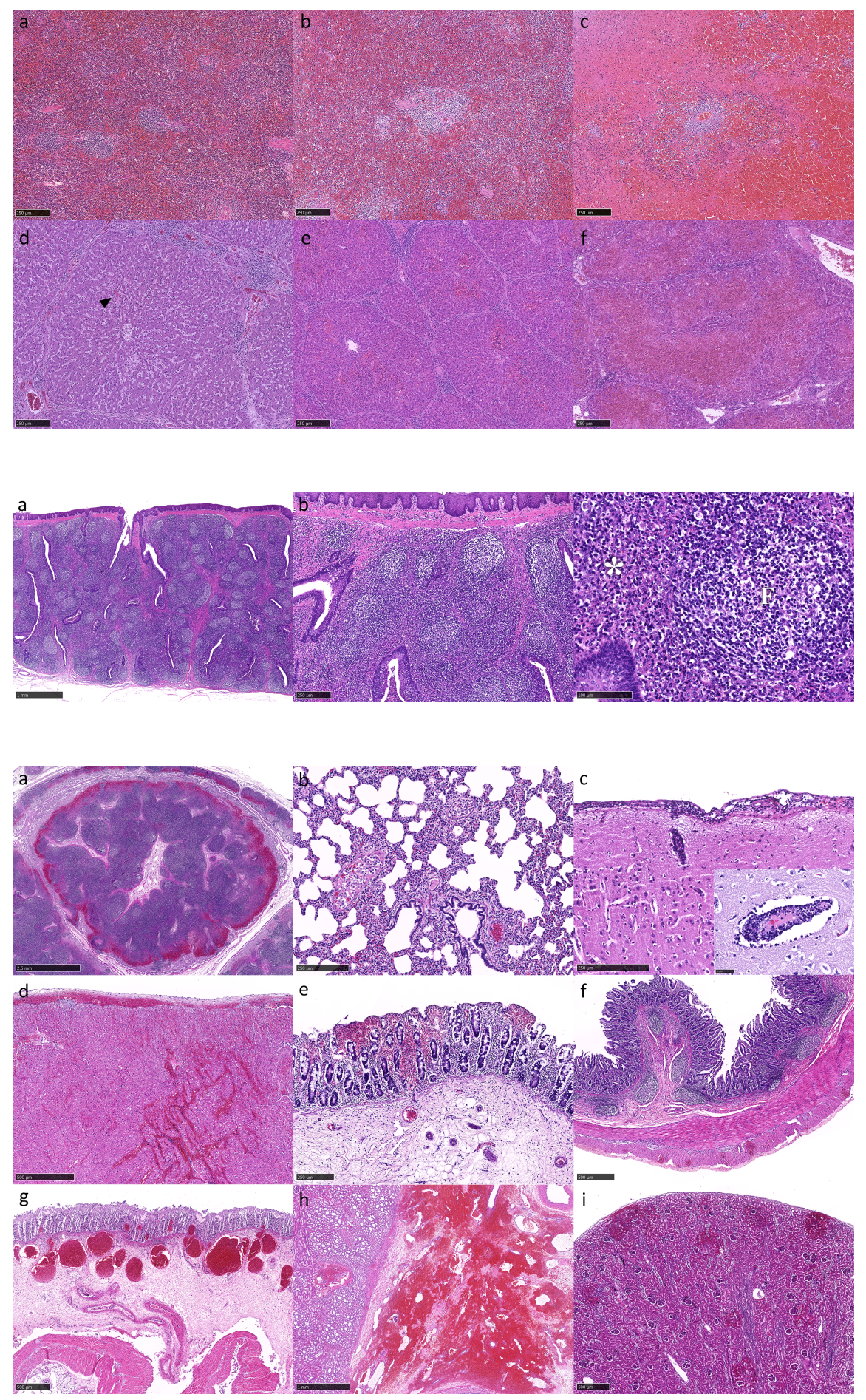


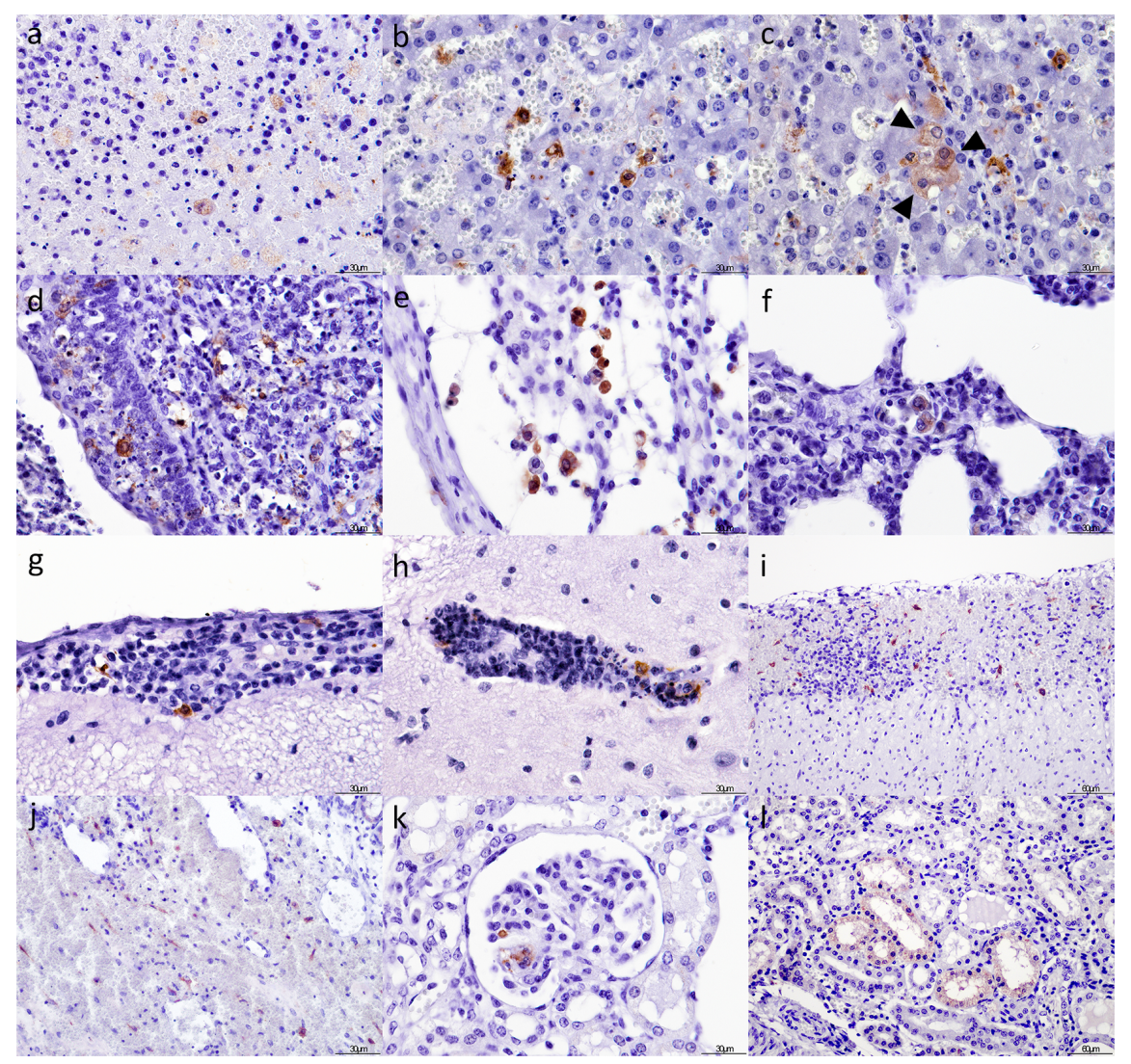

